\title{
Macro-mesoscale microstructural evolution modeling under hot forging of a Ti-17 alloy with a lamellar $(\alpha+\beta)$ starting microstructure
}

\author{
Hiroaki Matsumoto $^{\mathrm{a}}{ }^{*}{ }^{*}$, Kenta Yamanaka ${ }^{\mathrm{b}}$, Akihiko Chiba ${ }^{\mathrm{b}}$, Yoko Yamabe-Mitarai ${ }^{\mathrm{c}}$, Yoshio Itsumi \\ d \\ ${ }^{\mathrm{a}}$ Faculty of Engineering and Design, Kagawa University, ${ }^{\mathrm{b}}$ Institute for Materials Research, Tohoku \\ University, \\ ${ }^{\mathrm{c}}$ Research center for Structure Materials, NIMS, ${ }^{\mathrm{d}}$ Kobe steel \\ *matsu_h@eng.kagawa-u.ac.jp
}

\begin{abstract}
:
Microstructural conversion mechanisms under hot forging process (at temperatures ranging from 750 ${ }^{\circ} \mathrm{C}$ to $1050{ }^{\circ} \mathrm{C}$ and strain rates ranging from $10^{-3} \mathrm{~s}^{-1}$ to $1 \mathrm{~s}^{-1}$ ) of a Ti-5Al-2Sn-2Zr-4Mo-4Cr (Ti-17) alloy with a lamellar starting microstructure were experimentally identified in this work. After that, constitutive formulae for predicting the microstructural evolution were established followed by calculation using finite-element (FEM) analysis. In the $\alpha$ phase, a lamellae kinking is the dominant mode in the higher strain rate region and dynamic globularization frequently occurs at higher temperatures. On the other hand, continuous dynamic recrystallization is the dominant mode below the transition temperature, $\mathrm{T} \beta\left(880 \sim 890^{\circ} \mathrm{C}\right)$ in the $\beta$ phase. And, at conditions of lower strain rates and higher temperatures, dynamic recovery tends to be more active. For microstructural prediction, a set of constitutive equations modeling the microstructural evolution and forging properties are established by optimizing the experimental data followed by implementation in the DEFORM-3D software package. Herein, microstructural evolution on dynamic globularization process, dynamic recrystallization behavior are predicted according to both approaches of physical model and artificial neural network model followed by FEM simulation. In these calculated results, there is a satisfactory agreement between the experimental and simulated results, indicating that the established series of constitutive models can be used to reliably predict the properties of a Ti-17 alloy after forging.
\end{abstract}




\section{Introduction}

Ti-5Al-2Sn-2Zr-4Mo-4Cr (Ti-17) alloy, a near- $\beta$-type $(\alpha+\beta)$ alloy with high strength, superior fracture toughness, and excellent creep property, was first developed by GE Aviation,[1] which is being widely used to manufacture fan blades and compressor disks in aircraft engines. For hot deformation of metallic alloys, dynamic recovery (DRV) and dynamic recrystallization (DRX) occur and result in microstructural conversion. [2] Herein, the DRX processes include (1) discontinuous dynamic recrystallization (DDRX), in which new grains are formed by heterogeneous nucleation and growth processes, and (2) continuous dynamic recrystallization (CDRX), in which subgrains with lowangle boundaries are formed that subsequently evolve into grains with a high fraction of high-angle boundaries, with increasing plastic strain. It is well recognized that the DRV or CDRX is reported to be dominant for the hot deformation of near $\alpha$ and $(\alpha+\beta)$ Ti alloys. [3] Furthermore, under hot working of Ti alloys, dynamic microstructural changes such as grain size evolution, change in $\alpha$ phase fraction and dynamic globularization (DG) of $\alpha$ phase also occur. These microstructural evolutions strongly affect the mechanical properties of Ti-products. The dynamic microstructural changes, which occur during deformation, are dependent on strain rate, temperature, applied strain and material initial microstructure. [4] In order to explore and understand the deformation behavior, involved microstructural evolution and optimization of deformation process, a series of models for accurate microstructural prediction should be established. The present work focuses on the microstructural predictions (DG behavior of $\alpha$ phase and DRX behavior of $\beta$ phase) and prediction of a Vickers hardness for the forging of a Ti-17 alloy with a lamellar $(\alpha+\beta)$ starting microstructure. Initially, the microstructural conversion mechanism for the hot forging of the Ti-17 alloy with a lamellar $(\alpha+\beta)$ starting microstructure were examined through detailed microstructural observations and analysis of deformation kinetics according to Ref. [5]. Later, constitutive formulae on the physical model were established on the basis of the experimental results and the classical Johnson-Mehl-AvramiKolmogorov (JMAK) formulation which enables us to predict the DRX behavior of Ti alloys. [6-8] Additionally, this work also applied an artificial neural network (ANN) model to predict the microstructure and Vickers hardness. The ANN model cannot identify the deformation and microstructural evolution mechanisms, whereas it has a relatively high precision for prediction owing to the advanced statistical approach. These constitutive formulae of physical model and ANN model were implemented in the finite-element method (FEM) software (DEFORM-3D, v.10.2) followed by simulation of microstructures and Vickers hardness.

\section{Experimental procedure}

A Ti-17 alloy with a chemical composition (wt.\%) of Ti-4.89Al-2.00Sn-1.92Zr-3.82Cr-3.93Mo$(0.09 \mathrm{Fe}-0.014 \mathrm{Si}-0.102 \mathrm{O}-0.01 \mathrm{C})$ was used in this work. Initial microstructure before hot forging is a lamellar $(\alpha+\beta)$ microstructure as shown in Fig. 1(a). From Fig. 1(a), the black and white phases 
correspond to $\alpha$ phase and $\beta$ phase, respectively. Isothermal forging tests were performed at the process temperatures of $750{ }^{\circ} \mathrm{C}, 800{ }^{\circ} \mathrm{C}, 850{ }^{\circ} \mathrm{C}, 900{ }^{\circ} \mathrm{C}, 950{ }^{\circ} \mathrm{C}$, and $1050{ }^{\circ} \mathrm{C}$, and the strain rates ranging from $10^{-3} \mathrm{~s}^{-1}$ to $1 \mathrm{~s}^{-1}$. The specimens $(5 \mathrm{~mm}$ diameter and $7.5 \mathrm{~mm}$ height) used for the forging test were fabricated by electron discharge machining. The forging test was conducted at a constant strain rate and a height true strain of approximately 0.75 . The obtained stress-strain curves were corrected by the friction correction and adiabatic correction methods according to Refs. [9-11] in order to obtain accurate stress-strain curves. Microstructure was analyzed with a field emission scanning electron microscope (FE-SEM) fitted with an electron back-scattering diffraction (EBSD). The forging characteristics of the Ti-17 alloy with sizes of $\varphi 120 \mathrm{~mm}-\mathrm{h} 240 \mathrm{~mm}$ were analyzed by FEM with a DEFORM-3D software (v. 10.2) using a user-defined subroutine (for the physical and ANN models).

\section{Results and discussion}

\section{3-1. Microstructures before and after forging}

Figure 1 shows (a) the initial microstructure of a Ti-17 alloy before forging and the some typical deformed microstructures forged at (b) $750^{\circ} \mathrm{C}-10^{-3} \mathrm{~s}^{-1}$, (c) $850^{\circ} \mathrm{C}-10^{-1} \mathrm{~s}^{-1}$, (d) $850^{\circ} \mathrm{C}-10^{-3} \mathrm{~s}^{-1}$, (e) $1050^{\circ} \mathrm{C}-10^{-1} \mathrm{~s}^{-1}$ and (f) $1050^{\circ} \mathrm{C}-10^{-3} \mathrm{~s}^{-1}$. The microstructures are shown by SEM-BSE images, black and grey regions correspond to the $\alpha$ and $\beta$ phases, respectively. A fine $(\alpha+\beta)$ lamellar microstructure is seen in the starting microstructure [Fig. 1(a)]. Additionally, it is seen from the result of optical microscopy that the lamellar microstructure is formed in the coarse prior $\beta$ grained microstructure (with an average grain size of approximately $1 \mathrm{~mm}$ ). For forged specimens, we can observe from Fig. (b) for testing at $750{ }^{\circ} \mathrm{C}$ that a lamellae kinking behavior in which the $\alpha$ phase exhibits a bending is enhanced. The observed lamellae kinking results in a flow instability during deformation. For the forging at $850^{\circ} \mathrm{C}-10^{-1} \mathrm{~s}^{-1}$ and $10^{-3} \mathrm{~s}^{-1}$ as shown in Figs. $1(\mathrm{c})(\mathrm{d})$, the decrease in $\alpha$ fraction as compared to the initial microstructure and the enhanced dynamic globularization are observed. Herein, globularization is seen to be enhanced at the lower strain rate of $10^{-3} \mathrm{~s}^{-1}$ than at $10^{-1} \mathrm{~s}^{-1}$. The nucleation sites for globularization are at the kinks in the lamellae as well as at some of $\beta$ grain boundaries. [12] This type of microstructural conversion is recognized as the geometric dynamic recrystallization behavior. With respect to microstructural conversion of the $\beta$ phase, DRV or CDRX is a dominant mode. When the testing temperature is above $T_{\beta}\left(880 \sim 890^{\circ} \mathrm{C}\right)$, a heterogeneous $\beta$ grained microstructure composed of deformed coarse prior $\beta$-grains and fine equiaxed $\beta$-grains formed at the boundaries of the coarse $\beta$-grains is observed for the forged specimen tested at $1050^{\circ} \mathrm{C}-10^{-1} \mathrm{~s}^{-1}$ [Fig. $1(\mathrm{e})]$. On the other hand, a finer equiaxed $\beta$-grained microstructure with sizes less than the initial grain size of prior $\beta$ grains (approximately $1 \mathrm{~mm}$ ) is revealed from Fig. 1(f), indicating that CDRX is the dominant microstructural conversion mode in the $\beta$ phase at lower strain rate region. 
(a)

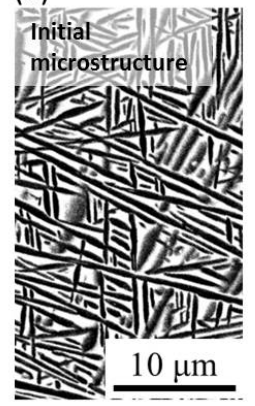

(d)

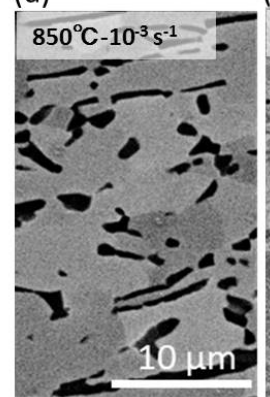

(b)

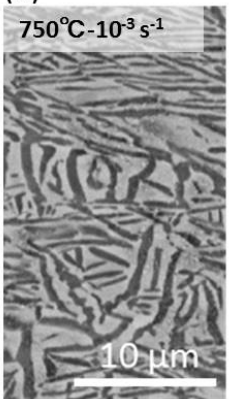

(e)

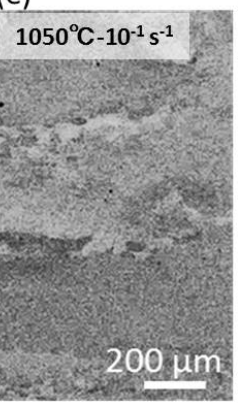

(c)

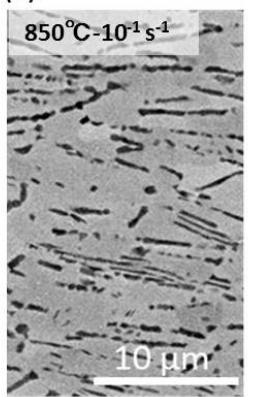

(f)

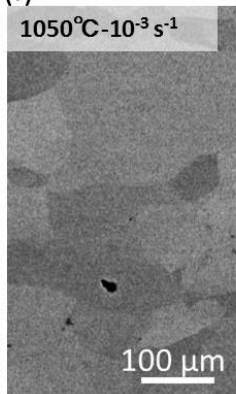

Fig. 1 SEM-BSE images showing (a) the initial microstructure with an $(\alpha+\beta)$ lamellar microstructure and the forged microstructures tested at (b) $750^{\circ} \mathrm{C}-10^{-3} \mathrm{~s}^{-1}$, (c) $850^{\circ} \mathrm{C}-10^{-1} \mathrm{~s}^{-1}$, (d) $850^{\circ} \mathrm{C}-10^{-3} \mathrm{~s}^{-1}$, (e) $1050^{\circ} \mathrm{C}-10^{-}$ $1 \mathrm{~s}^{-1}$ and (f) $1050^{\circ} \mathrm{C}-10^{-3} \mathrm{~s}^{-1}$.

\section{3-2. Flow behavior under forging}

Figure 2 shows the true stress- true strain curves of (a) the $(\alpha+\beta)$ region $\left(750^{\circ} \mathrm{C} \sim 850^{\circ} \mathrm{C}\right)$ and (b) the $\beta$ region $\left(900^{\circ} \mathrm{C} \sim 1050^{\circ} \mathrm{C}\right)$. Herein, the corrected curves are expressed by dotted lines. The corrections of friction and increasing temperatures were carried out according to the reports [9-11]. From Fig. 2, extensive flow softening is clearly observed at higher strain rates in the $(\alpha+\beta)$ region, while almost steady state flow behaviors are seen for the forged specimens at lower strain rates in the $(\alpha+\beta)$ region and for the forged specimens in the $\beta$ region. Additionally, a quite dissimilar stress value before and after correction is revealed especially at higher strain rates in the $(\alpha+\beta)$ region [Fig. 2(a)], which is mainly due to the effect of adiabatic heating during the deformation at high strain rates. A lamellae kinking observed for forged specimen at high strain rates in the $(\alpha+\beta)$ region is also deduced to affect the extensive flow softening (resulting in flow instability). According to the results of microstructure, a steady state stress behavior observed in forging conditions at higher temperatures and lower strain rates is attributable to dominant microstructural conversion mode by either DRV or CDRX of $\beta$ phase. 
(a)

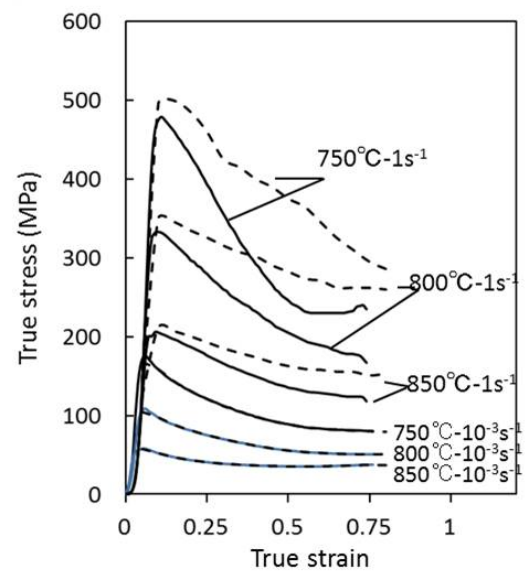

(b)

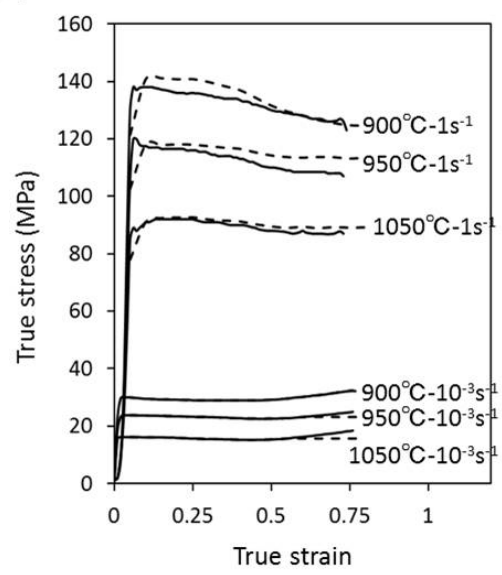

Fig. 2 True stress - true strain curves (experimentally obtained curves and corrected curves) of the Ti-17 alloy tested in (a) $(\alpha+\beta)$ region and (b) $\beta$ region.

\section{3-3. Microstructural conversion mode}

Figure 3 shows the relationship between the DRX $\beta$-grain size and Zener-Hollomon $(Z)$ parameter (in $\log$ scale $)$ in the $(\alpha+\beta)$ and $\beta$ regions. Here, $Z$ parameter is expressed as shown in equation (1)

$$
Z=\dot{\varepsilon} \exp (Q / R T)
$$

where $R$ is the universal gas constant $[8.314 \mathrm{~J} /(\mathrm{mol} \cdot \mathrm{K})], T$ is the absolute temperature, and $Q$ is the apparent activation energy for deformation. Here, the $Q$ are $365.4 \mathrm{~kJ} / \mathrm{mol}$ [in $(\alpha+\beta)$ region] and 172.8 $\mathrm{kJ} / \mathrm{mol}$ (in $\beta$ region), respectively. From Fig. 3, linear relationships are observed clearly, indicating that the grain size itself is dependent on the thermally activated controlling the fine grain formation.

Figure 4 shows the summary of microstructural conversion mode of (a) the $\alpha$ phase and (b) the $\beta$ phase after forging at a height true strain of approximately 0.75 . For the $\alpha$ phase, a lamellae kinking is dominant mode in the higher strain rate region and dynamic globularization is more enhanced with increasing temperature. On the other hand, from Fig. 4(b) showing the result of $\beta$ phase, CDRX is dominant mode below the $T_{\beta}$, and DRV is further enhanced with increasing temperature (above $T_{\beta}$ ) and strain rate. That is, for the forging above $T_{\beta}, \mathrm{CDRX}$ frequently occurs especially at lower strain rate region. 


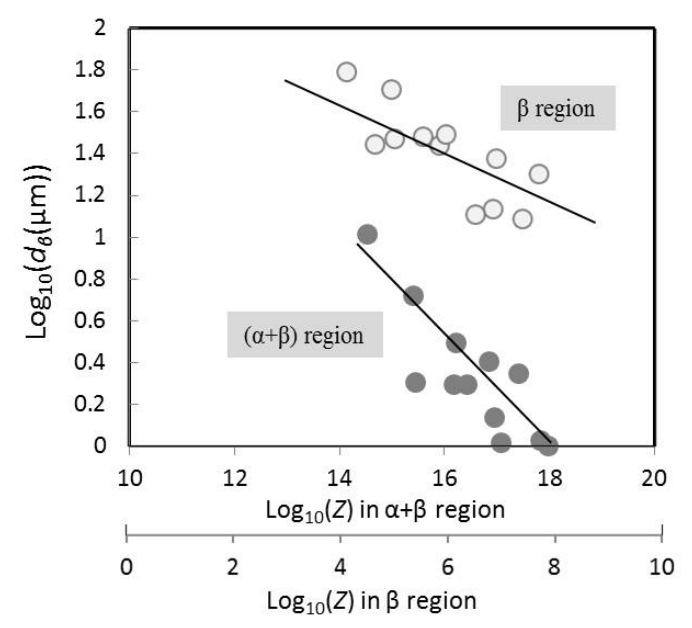

Fig. 3 Relationship between the DRX grain size of $\beta$ phase and $Z$ parameter in $\log -\log$ scale.
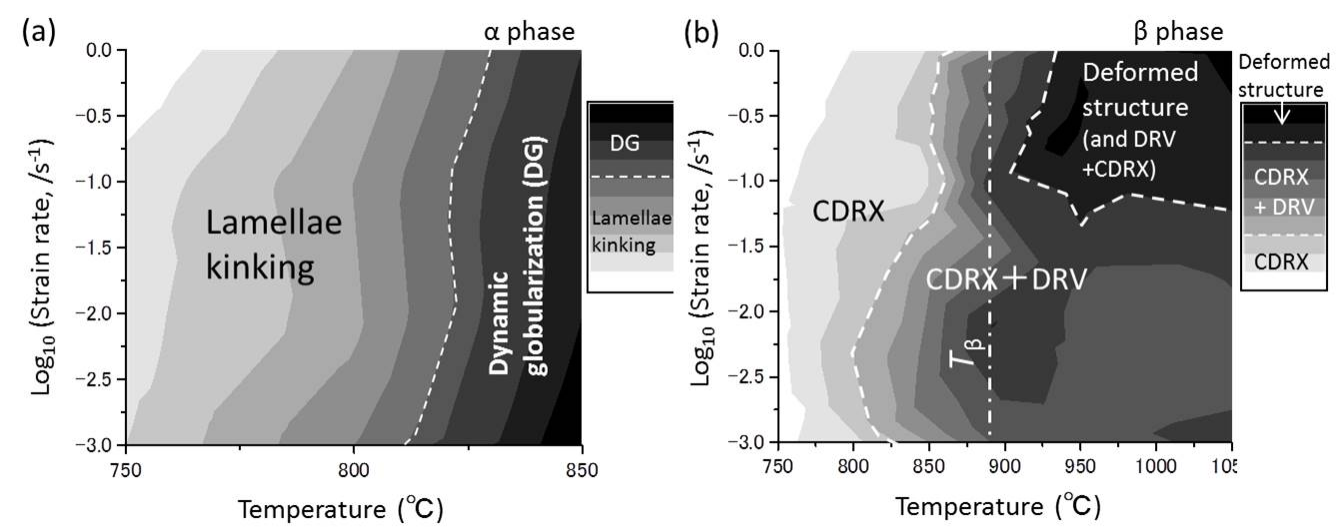

Fig. 4 Microstructural evolution modes experimentally determined of (a) $\alpha$ phase and (b) $\beta$ phase as functions of temperature and strain rate.

\section{3-4. Prediction of microstructure and hardness according to physical modeling and neural network}

In this study, the characteristics of dynamic globularization behavior of $\alpha$ phase and DRX of $\beta$ phase are described by physical model of Johnson-Mehl-Avrami-Kolmogorov (JMAK) equation. [13]

For dynamic globularization fraction $\left(f_{D G}\right)$ of the $\alpha$ phase, it is defined as follows 


$$
f_{D G}=1-\exp \left[-k_{D G} \times\left(\varepsilon-\varepsilon_{D G}\right)^{n_{D G}}\right]
$$

where $f_{D G}$ is the dynamic globularization fraction, $k_{D G}$ and $n_{D G}$ are material constants, $\varepsilon$ is the true strain, and $\varepsilon_{C}$ is the critical strain for the onset of dynamic globularization. The $\varepsilon_{C}$ is calculated as

$$
\varepsilon_{D G_{-} C}=0.0149 \exp \left(49.27 \cdot \varepsilon_{D G_{-} P}\right)
$$

in which $\varepsilon_{D G_{-} P}$ corresponds to the true strain at peak stress in flow curve (which is experimentally identified) and expressed as follows

$$
\varepsilon_{D G_{-} P}=0.0035 \cdot Z^{0.0782}
$$

where $Z$ is the Zener-Hollomon parameter as expressed in Eq.(1).

The parameter $k_{D G}$ (in Eq.(2)), which denotes the reaction rate, is calculated using the following equation, in which the effect of strain rate has been considered.

$$
k_{D G}=k_{D G 0} \dot{\varepsilon}^{m_{1}} \exp \left(-\frac{Q_{1}}{R T}\right)
$$

On the other hand, the DRX behavior of $\beta_{\mathrm{d}}$ phase according to JMAK equation is as follows:

$$
f_{D R X}=1-\exp \left[-\beta_{d} \times\left(\frac{\varepsilon-\varepsilon_{D R X_{-}}}{\varepsilon_{D R X_{-}}}\right)^{n_{D R X}}\right]\left(\varepsilon \geq \varepsilon_{D R X_{-} c}\right)
$$

where $f_{D R X}$ is the DRX fraction, $b_{d}$ and $n_{D R X}$ are material constants, $\varepsilon$ is the true strain, and $\varepsilon_{D R X \_} c$ is the critical strain for DRX initiation. The $\varepsilon D R X \_0.5$ is calculated as:

$$
\begin{aligned}
& \varepsilon_{D R X_{-} 0.5}=a_{1} d_{0}{ }^{n 1} \dot{\varepsilon}^{m 1} \exp \left(\frac{Q_{2}}{R T}\right) \\
& \beta_{d}=\beta_{D R X_{-} 0} \exp \left(\frac{Q_{3}}{R T}\right)
\end{aligned}
$$


where $\dot{\varepsilon}$ is the strain rate, and $a_{1}, n_{1}, m_{1}, n_{D R X}, Q_{2}, Q_{3}$, and $\beta_{D R X \_} 0$ are material constants. The term $d_{0}(\mathrm{~mm})$ is the initial grain size before deformation. The critical strain $\left(\varepsilon_{D R} X_{-}\right)$is determined by the peak strain $\left(\varepsilon D R X_{p} p\right)$ (which is experimentally identified) as follows:

$$
\varepsilon_{D R X_{-} c}=0.8 \times \varepsilon_{D R X_{-} p}
$$

The material constants in the above equations were determined on the basis of the minimization of the sum of errors between the experimental and the calculated data. Herein, the material constants were optimized and determined by non-linear regression analysis.

The microstructural evolution model as stated above has been implemented into the FEM software of DEFORM-3D (v.10.2) through a user-subroutine followed by simulation of isothermal forging. Here, the incremental form of the equation was developed expressed in Eq. (10) to Eq. (13). Thanks to this incremental form, it enables to accumulate small changes of temperatures and strain rate at each step in FEM analysis during forging process.

$$
\begin{gathered}
f_{D G}{ }^{n+1}=f_{D G}{ }^{n}+d\left(f_{D G}\right)^{n} \\
d\left(f_{D G}\right)^{n}=\frac{\partial f}{\partial \varepsilon} d \varepsilon+\frac{\partial f}{\partial \varepsilon_{D R X_{-} c}} d \varepsilon_{D R X_{-} c}+\frac{\partial f}{\partial k_{D G}} d k_{D G} \\
f_{D R X}{ }^{n+1}=f_{D R X}{ }^{n}+d\left(f_{D R X}\right)^{n}(12) \\
d f_{D R X}{ }^{n}=\frac{\partial f_{D R X}}{\partial \varepsilon} d \varepsilon+\frac{\partial f_{D R X}}{\partial \varepsilon_{D R X_{-} 0.5}} d \varepsilon_{D R X_{-} 0.5}+\frac{\partial f_{D R X}}{\partial \varepsilon_{D R X_{-} C}} d \varepsilon_{D R X_{-} C}+\frac{\partial f_{D R X}}{\partial \beta_{d}} d \beta_{d}
\end{gathered}
$$

On the other hand, the artificial neural network (ANN) is an information treatment system with the characteristics of adaptive learning, which is suitable for treating non-linear phenomena and complex relationships. [14] The ANN is essentially an operation linking input to output data, by using a particular set of non-linear basis functions. The advantage of ANN is their ability to learn or adapt to changing conditions. The architecture of the ANN refers to the number of the layers in the ANN and the number of the neurons in each layer. This work set the one or two layers and three neurons in each layer for ANN calculation. Figure 5 (a) represents the schematic structure of ANN in this work. Here, DG fraction of $\alpha$ phase, DRX fraction of $\beta$ phase and Vickers hardness for the forged specimen are calculated through the ANN model with the input data of temperature, effective strain rate, effective strain and cooling rate after forging.

Figure 5(b)(c)(d) summarizes the comparison between the experimental data and the calculated data (according to physical model and ANN model) of (b) DG fraction of $\alpha$ phase in the forged specimens in $(\alpha+\beta)$ region and (c) DRX fraction of $\beta$ phase in the forged specimen in $\beta$ region and (d) Vickers hardness of the forged specimen. For both predicted results of physical model and ANN model, we 
can observe good correlation between the experimental and predicted results. Relatively, it can be noted that the predicted result according to the ANN model exhibits more close value with the experimental result. This result indicates that both models of the physical and the ANN approaches are able to predict the microstructures and hardness successfully.

(a)

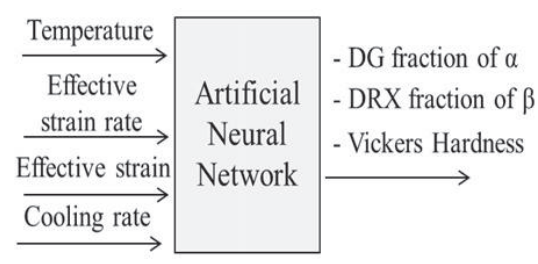

(c)

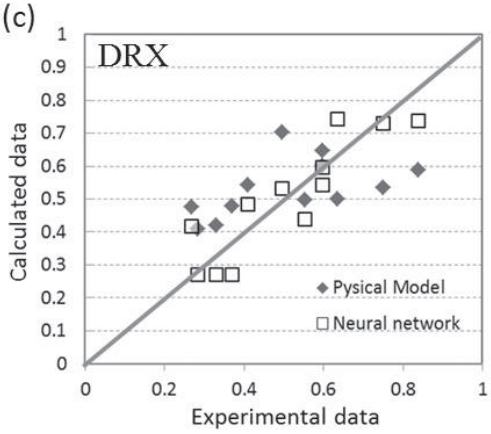

(b)

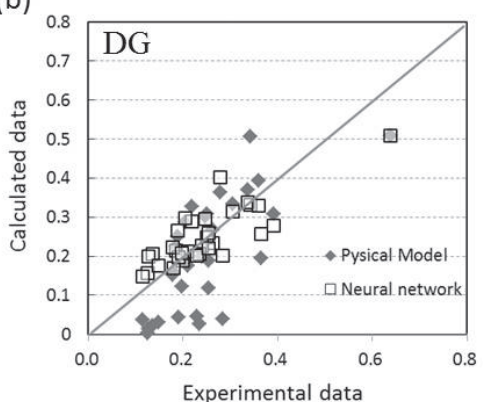

(d)

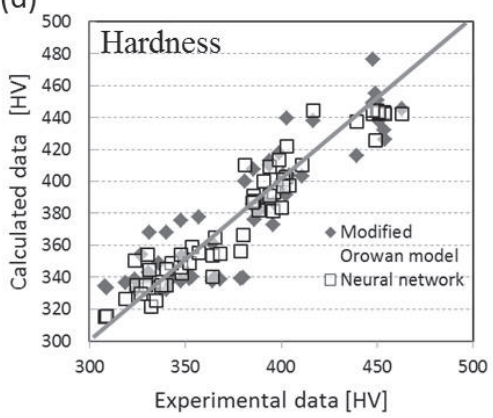

Fig. 5 (a) Architecture of the ANN model and comparison of calculated data (for the physical and ANN models) and experimental data of (b) dynamic globularization fraction of $\alpha$ phase [obtained in $(\alpha+\beta)$ region], (c) dynamic recrystallization fraction of $\beta$ phase (obtained in $\beta$ region) and (d) Vickers hardness.

The physical models established in this work and the ANN model were implemented in the DEFORM-3D (ver.10.2) FEM software through a user-defined subroutine followed by the simulation of isothermal forging. Figure 6 shows the distributions of (a)(b) DG fraction of $\alpha$ phase, (c)(d) DRX fraction of $\beta$ phase and (e) Vickers hardness according to calculation by (a)(c) physical model and (b) (d)(e) ANN model for the forged specimen across the cross-section. Here, forging conditions are (a)(b) $800^{\circ} \mathrm{C}-0.03 \mathrm{~s}^{-1},(\mathrm{c})(\mathrm{d})(\mathrm{e}) 910^{\circ} \mathrm{C}-0.03 \mathrm{~s}^{-1}$ and a height reduction of $75 \%$. We can observe from the heterogeneous distributions in Figs. 6(a)-(d) that DG and DRX are locally enhanced especially in the vicinity of central region. Such heterogeneous distributions of the forging property are also observed with respect to the effective strain, the effective strain rate and temperature, resulting in the 
heterogeneity of microstructures and Vickers hardness as shown in Fig. 6. From the distribution of Vickers hardness [Fig. 6(e)], a higher hardness is observed in the dead-zone region (corresponding to upper region in forged specimen) than in the central region where DRX of $\beta$ phase is enhanced. This result is attributable to the difference in $\alpha$ precipitation behavior under cooling, which is dependent on location of the forged specimen. Thus, mechanical property (Vickers hardness focused in this work) exhibits complicated heterogeneous distribution which is owing to the difference in microstructural conversion behaviors (DRX of $\beta$ phase and $\alpha$ precipitation) depending on location of forged specimen. Compared to the distributions of physical model results and ANN results from Figs. 6(a)-(d), we can observe the wider region that the fractions of DG and DRX exhibit high for the distributions of physical model. As abovementioned for Fig. 5, the ANN model result exhibits more close value with the experimental result, pointing out that modification of constitutive physical model is further required for an accurate prediction. During the hot deformation of near $\alpha$ and $(\alpha+\beta)$ Ti alloys, DRV or CDRX is reported to be dominant.[3] The constitutive physical model in this work followed JMAK law that was suitable for discontinuous typed behavior. Therefore, for additional, highly accurate prediction of the microstructure, a further direction of this study will be to focus on the modification of constitutive physical models considering the continuous typed microstructural evolution mode and the appropriate combination of the physical and ANN models.

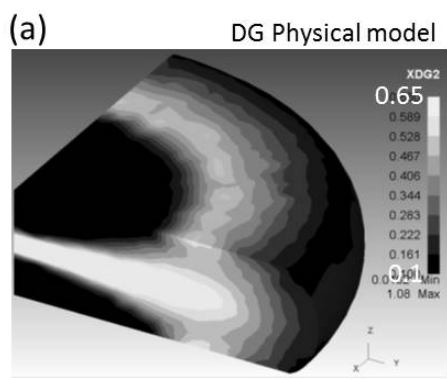

(d)

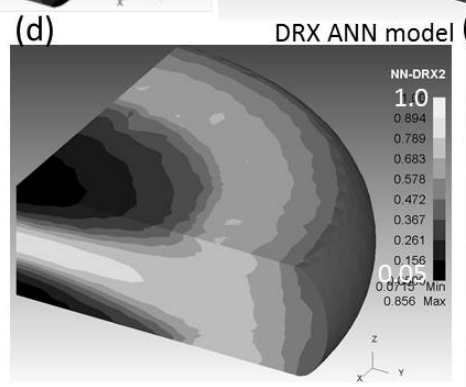

DG ANN model (c)
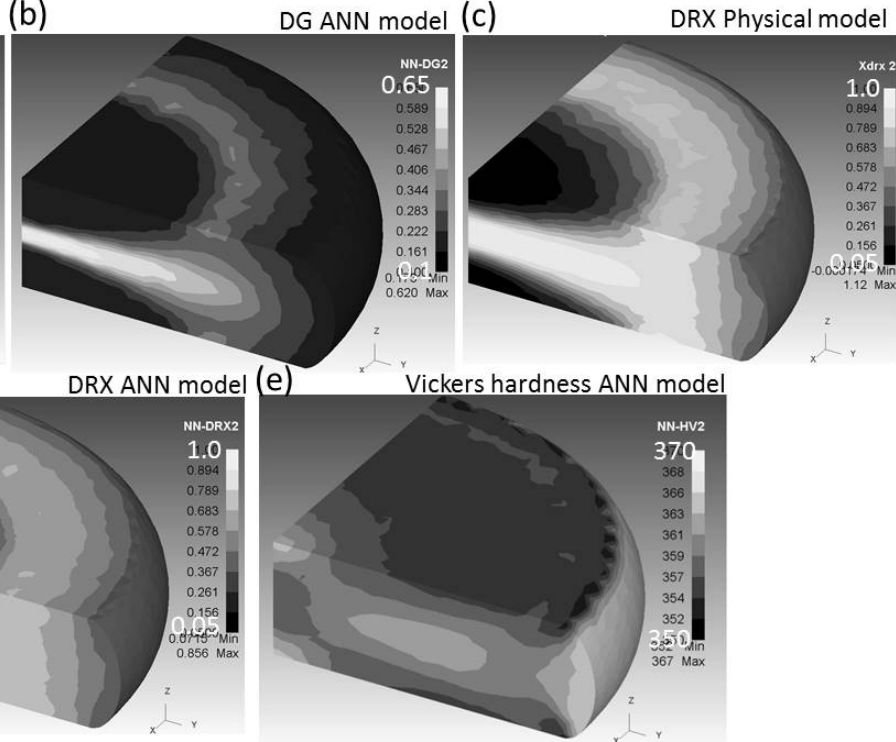

Fig. 6 Distributions from the FEM analysis for (a)(b) dynamic globularization fraction of $\alpha$ phase, (c)(d) dynamic recrystallization fraction of $\beta$ phase and (e) Vickers hardness according to (a)(c) the physical 
model and (b)(d)(e) the ANN model. Forging conditions are at (a)(b) $800^{\circ} \mathrm{C}-0.03 \mathrm{~s}^{-1}$ and (c)(d)(e) $910^{\circ} \mathrm{C}-0.03 \mathrm{~s}^{-1}$, respectively.

\section{Conclusions}

The microstructural evolution under hot forging of the Ti-17 alloy reveals that lamellae kinking is the dominant mode for the $\alpha$ phase at higher strain rates; dynamic globularization of the $\alpha$ phase frequently occurs at high temperatures. Regarding the $\beta$ phase, CDRX is the dominant mode at temperatures below $T_{\beta}$. Herein, DRV also occurs at lower strain rates. At testing temperatures above $T_{\beta}$, deformation associated with the occurrence of DRV is exhibited, while CDRX also frequently occurred with decreasing the strain rate.

The behaviors of dynamic globularization of $\alpha$ phase and dynamic recrystallization of $\beta$ phase were modeled into constitutive formulae expressed in terms of the JMAK equation followed by implementation in FEM analysis. Additionally, the microstructural prediction according to an ANN model was also carried out. It is found that both approaches of the physical and ANN models provide an approximate agreement with the experimental results. Thus, this work indicates the possibility of a reliable prediction of microstructures and mechanical property of the forged Ti-17 alloy.

\section{Acknowledgement:}

This work was supported by the Council for Science, Technology, and Innovation (CSTI), the Crossministerial Strategic Innovation Promotion Program (SIP), and the "Process Innovation for Super Heat-Resistant Metals (PRISM)" (Funding agency:JST).

\section{References}

[1] R.R. Boyer, H.W. Rosenberg, Beta Titanium Alloys in the 1980's. Atlanta. 1983 Mar:1984: 239.

[2] R.D. Doherty, D.A. Hughes, F.J. Humphreys, J.J. Jonas, D. Juul Jensen, M.E. Kassner, W.E. King, T.R. McNelley, H.J. McQueen, A.D. Rollett, Mater. Sci. Eng. A238 (1997) 219-274.

[3] I. Weiss, S.L. Semiatin, Mater. Sci. Eng. A 263 (1999) 243-256.

[4] C.M. Sellars, Mater. Sci. Technol. 15 (1990) 1072-1081. 
[5] H. Matsumoto, D. Naito, K. Miyoshi, K. Yamanaka, A. Chiba, Y. Yamabe-Mitarai, Sci. Tech. Adv. Mater. 18(1) (2017) 893-904.

[6] G.Z. Quan, G.C. Luo, J.T. Liang, et al., Comput. Mater. Sci. 97 (2015) 136-147.

[7] D.L. OuYang, M.W. Fu, S.Q. Lu, Mater. Sci. Eng. A 619 (2014) 26-34.

[8] K.L. Wang, M.W. Fu, S.Q. Lu, et al., Mater. Design 32 (2011) 1283-1291.

[9] Y. Li, E. Onodera, A. Chiba, Mater. Trans. 51 (2010) 1210-1215.

[10] Y. Li, E. Onodera, H. Matsumoto, et al., ISIJ Int. 51 (2011) 782-787.

[11] M. Mataya, V.E. Sackschewsky, Metall. Mater. Trans. A 25 (1994) 2737-2752.

[12] S.L. Semiatin, V. Seetharaman, I. Weiss, Mater. Sci. Eng. A 263 (1999) 257-271.

[13] C.M. Sellars, J.A. Whiteman, Metal Sci. 13 (1979) 187-194.

[14] D.E. Rumelhart, G. Hinfon, R. Williams, Nature 323 (1986) 533-536. 